\title{
REFORMA DE SALUD EN ECUADOR: NUNCA MÁS EL DERECHO A LA SALUD COMO UN PRIVILEGIO
}

\author{
Miguel Malo-Serrano, ${ }^{1, a}$, Nicolás Malo-Corral ${ }^{2, b}$
}

\begin{abstract}
RESUMEN
El proceso de reforma de la salud que experimenta el Ecuador ha tenido logros importantes, porque se da en el marco de una nueva Constitución de la República, que permitió la incorporación de demandas sociales históricas surgidas de las críticas al neoliberalismo en la reestructuración y modernización estatal. Los ejes de la reforma consisten en tres componentes: por un lado, en organizar un Sistema Nacional de Salud que supere la fragmentación anterior, que constituya la Red Pública Integral de Salud; políticas encaminadas a fortalecer la atención primaria de salud, articulando la acción sobre los determinantes de la salud; $y$, por último, el incremento del financiamiento para consolidar las transformaciones. Concluimos que los desafíos en la reforma tienen que ver con la sustentabilidad de los procesos, sostenibilidad financiera del sistema y con la mayor activación de mecanismos de participación que permitan la veeduría ciudadana de los servicios y el empoderamiento de los ciudadanos de su derecho a la salud.
\end{abstract}

Palabras clave: Reforma de la atención de salud, Ecuador; Atención primaria de salud; Financiación de la atención de la salud (fuente: DeCS BIREME).

\section{HEALTH REFORM IN ECUADOR: NEVER AGAIN THE RIGHT TO HEALTH AS A PRIVILEGE}

\begin{abstract}
The process of the health reform being experienced by Ecuador has had significant achievements because it occurs within the framework of a new Constitution of the Republic, which allowed the incorporation of historical social demands that arose from the criticism of neoliberalism in the restructure and modernization of the state. The backbone of the reform consists of three components: organization of a National Health System that overcomes the previous fragmentation and constitutes the Integral Public Health Network; development of policies to strengthen primary health care, articulating actions on the determinants of health, and finally, increasing funding to consolidate these changes. We conclude that challenges to the reform are related to the sustainability of the processes, financial sustainability of the system, greater activation of participatory mechanisms that enable citizen assessment of services and citizen empowerment regarding their right to health.
\end{abstract}

Key words: Health care reform, Ecuador; Primary health care; Healthcare financing (source: MeSH, NLM).

\section{INTRODUCCIÓN}

Tras años de inestabilidad política, ocho cambios presidenciales en una década, e inestabilidad económica, crisis financiera y posterior dolarización de la economía, la elección del presidente Correa en el año 2006, se da en el contexto de las reacciones de los movimientos sociales ante las reformas neoliberales de los 90 e inicios de siglo (1) y las reivindicaciones sociales por una renovación de la clase política. Este nuevo gobierno, llamado de "la Revolución Ciudadana", significó el inicio de una etapa de estabilidad democrática que rescata el rol del Estado en la garantía y promoción del goce de los derechos humanos para toda la población. No sería posible analizar el proceso de reforma del sistema de salud de Ecuador sin ubicarlo apropiadamente en este contexto, que implica un marco jurídico, ético y político que la hace posible, y del cual destacaríamos lo siguiente.

\section{LA SALUD Y SUS DETERMINANTES EN LA CONSTITUCIÓN}

En la Constitución del 2008, no solamente se ratifica la salud como un derecho y la obligación del Estado para garantizarla, sino que por primera vez en este

\footnotetext{
Ministerio de Salud Pública del Ecuador. Quito, Ecuador.

Ministerio de Inclusión Económica y Social. Quito, Ecuador.

a Doctor en Medicina, maestría en Salud Pública; ${ }^{\text {b }}$ sociólogo con mención en desarrollo.

Recibido: : 30-09-14 Aprobado: 19-11-14
}

Citar como: Malo-Serrano M, Malo-Corral N. Reforma de salud en Ecuador: nunca más el derecho a la salud como un privilegio. Rev Peru Med Exp Salud Publica. 2014;31(4):754-61. 
nivel de marco jurídico se explicita que ese derecho a la salud no puede estar garantizado si no se garantizan otros derechos, es decir, el reconocimiento de la salud dependiente de determinantes fuera del sector. EI artículo 32 de la Carta Magna expresa: La salud es un derecho que garantiza el Estado, cuya realización se vincula al ejercicio de otros derechos, entre ellos el derecho al agua, la alimentación, la educación, la cultura física, el trabajo, la seguridad social, los ambientes sanos y otros que sustentan el buen vivir (2).

\section{LA INTERSECTORIALIDAD COMO UN REQUISITO PARA LA IMPLEMENTACIÓN DE LAS POLÍTICAS PÚBLICAS}

La acción del gobierno está orientada por el "Plan Nacional del Buen Vivir", cuya implementación exige mecanismos de gestión para un abordaje intersectorial de los problemas, pues sus objetivos no son sectoriales, pero requieren la articulación de las políticas sectoriales para su cumplimiento. Por ejemplo, el cumplimiento de las metas en la erradicación de la desnutrición crónica en niños menores de 2 años compete no solo a las autoridades de Salud, sino también de Educación, Inclusión Económica y Social, Agricultura, coordinadas por el Ministerio Coordinador de Desarrollo Social. De manera que la arquitectura institucional del Estado a través de los ministerios coordinadores propicia la intersectorialidad. Todo esto ha permitido, en la práctica de la gestión estatal, articular la "salud en todas la políticas" (3).

\section{LA RECUPERACIÓN DEL ROL DEL ESTADO}

En la Constitución se destaca la rectoría del Estado en los asuntos de interés público. La gestión del gobierno de la "Revolución Ciudadana" ha significado una dura lucha para recuperar la institucionalidad del Estado, que se vio muy disminuida como producto de las políticas neoliberales. Uno de los indicadores de esta recuperación ha sido su política económica soberana que, a través de renegociación de la deuda externa; renegociación de los contratos petroleros, y el enorme incremento en la recaudación de impuestos, ha permitido aumentar de manera significativa los ingresos fiscales para orientarlos a la inversión social. De hecho, el nuevo marco constitucional ha requerido la reforma y creación de leyes orientadas a cumplir su mandato hacia el buen vivir y modernizar el Estado, fortaleciendo su rectoría y haciéndolo más eficiente. Con la aprobación de más de 170 leyes ${ }^{(4)}$, en los últimos años se ha transformado profundamente el funcionamiento del Estado ecuatoriano y el rumbo de sus políticas.

\section{LA JUSTICIA SOCIAL: PRIORIDAD DE LA POLIITICA PÚBLICA}

Ciertamente, lo que ha marcado la diferencia en la mejora de la calidad de vida en el Ecuador ha sido la redistribución de la riqueza con una fuerte inversión social. A partir del 2007, el Estado ecuatoriano cambió sus prioridades, destinando más recursos a la inversión social que al pago de la deuda. En el 2013, el Ecuador invirtió 4,5 veces más en gasto social que en el 2006, año en el que la inversión pública representaba el 4,2\% del PIB, mientras que para el 2012 la cifra se triplicó, representando el $12,6 \%{ }^{(5)}$. Lo que implica pasar de un per cápita de inversión social de 147 USD en el 2006 a 446 USD en el 2011 (6). Producto de esta inversión, algunos indicadores reflejan importantes avances hacia la justicia social. En lo que se refiere al Coeficiente de Gini, del 2000 al 2006 este indicador solo se redujo en $2,9 \%{ }^{(7)}$, mientras que entre el 2006 al 2014 se redujo en $9,8 \%$.

La duplicación del salario básico desde el 2007, sumado a otros beneficios sociales, permitirá al 2014 que esta cubra el costo de la canasta básica familiar. El desempleo pasó de $6,47 \%$ en el 2009 a $4,15 \%$ en el 2013. Igualmente, el porcentaje de personas ocupadas en el subempleo disminuyó del $58,7 \%$ en el 2007 al $52,49 \%$ en el $2013{ }^{(8)}$. Para el año 2006 el $37,6 \%$ de la población vivía en situación de pobreza y el 16,9\% en pobreza extrema; actualmente, el $24,5 \%$ de la población vive en situación de pobreza y $8 \%$ en pobreza extrema. Cerca de un millón cincuenta mil personas han salido de la pobreza y más de 800 mil de la pobreza extrema ${ }^{(9)}$.

\section{REFORMA DE LA SALUD EN ECUADOR}

\section{ANTECEDENTES}

El inicio del gobierno de Correa se encuentra con un sector salud que presenta las consecuencias de un prolongado deterioro producto de las políticas neoliberales de los años 90 y de la inestabilidad democrática de inicios del 2000, con la reducción progresiva del presupuesto de salud, mecanismos llamados de autogestión que implicaban cobro directo por los servicios al usuario, traspaso sin financiamiento de servicios para municipios, falta de inversión en infraestructura, en recurso humano y pérdida de rectoría, que daba como resultado baja cobertura y 
calidad de los servicios públicos, con el consecuente aumento de gasto de bolsillo, que afectó principalmente a la población más pobre ${ }^{(10)}$. La intervención en el sector Salud implicaba, entonces, el rescate de una institución en profunda crisis, que ha requerido dos condiciones indispensables: un enorme esfuerzo fiscal para tratar de cerrar las brechas de calidad y accesibilidad en la atención, y gran firmeza política para recuperar la gobernabilidad del sector, reformando los marcos normativos e institucionales para rescatar la autoridad sanitaria del Estado y particularmente del Ministerio de Salud Pública (MSP).

\section{INVERSIÓN: DE LO MÍNIMO A LO NECESARIO}

La enorme inversión ha significado la adecuación, ampliación y renovación integral de toda la red hospitalaria en su infraestructura y equipamiento, así como el proceso de construcción de 18 nuevos hospitales, de los cuales 6 se finalizaron en el 2013. Esta inversión ha sido orientada también a garantizar de manera equitativa la atención prehospitalaria, reduciendo la tasa de 115000 a 35000 habitantes por ambulancia (11), y su vinculación al Servicio Integrado de Seguridad (SIS) ECU-911 que gestiona en todo el territorio la atención de las situaciones de emergencia (12). A partir del 2012, una vez establecida la planificación territorial coordinada de la Red Pública Integral de Salud (RPIS), y los estándares para los centros de salud de primer nivel, se ha iniciado su construcción para completar un total de 171 nuevos hasta el $2017^{(13)}$.

Además del incremento correspondiente del gasto corriente para cubrir el aumento salarial y la ampliación de personal, un rubro fuerte de inversión ha sido destinado a la formación del recurso humano. El Estado ecuatoriano ha entregado más de 2750 becas para los diferentes profesionales de salud (14). Igualmente, en medicamentos el rubro de gasto ha crecido de poco más de 100 millones en el 2007 a más de 380 millones en el 2013. Toda esta inversión ha significado mejorar drásticamente el acceso de la población a los servicios públicos. En el 2006 se registraron 14372251 atenciones de salud, mientras que para el año 2013 fueron $38088410{ }^{(15)}$.

\section{RECTORÍA DEL MINISTERIO DE SALUD PÚBLICA: ASEGURANDO LA GOBERNABILIDAD DESDE EL INTERÉS PÚBLICO}

La definición político-estratégica de la reforma es orientarse a la construcción de un Sistema Nacional de Salud (SNS) que tiene como elemento clave la existencia de una autoridad sanitaria nacional, cuya rectoría no solamente se refiere a la prestación de servicios de salud, sino también al que hacer sobre los determinantes de la salud. Autoridad sanitaria nacional que debe configurarse como una estructura de Estado que garantice en el largo plazo la prevalencia del interés público sobre el privado. Esto ha implicado un proceso de rescate de la rectoría del sector por parte del MSP que se ha desarrollado en dos aspectos: la adecuación del marco jurídico-normativo, y en la institucionalidad que permita sostener e implementar ese marco normativo. Estos dos aspectos se materializan en los ejes de la reforma que se describen a continuación.

\section{RED PÚBLICA INTEGRAL DE SALUD}

El sistema de salud ecuatoriano, como muchos de los países de nuestra región, ha sido un sistema fragmentado compuesto por servicios de salud públicos del MSP, del Instituto Ecuatoriano de Seguridad Social (IESS), Instituto de Seguridad Social de la Policía (ISSPOL), Instituto de Seguridad Social de las Fuerzas Armadas (ISSFA) y algunos servicios de menor cobertura de gobiernos locales y otros ministerios, cada uno funcionando de manera autónoma, con su propio sistema de financiamiento, política de recursos humanos, compras, etc. Además de la existencia de una oferta de servicios privados cuyo crecimiento ha sido directamente proporcional al deterioro de los públicos. Si bien, en inicios de los 2000 hubo algunos avances hacia la construcción del SNS, como La Ley del Sistema Nacional de Salud (2002) (16) y luego la Ley Orgánica de Salud en el $2006{ }^{\left({ }^{(17)}\right.}$, sin el respaldo político necesario estos marcos jurídicos tuvieron muy baja implementación. Luego, en el 2008, la propia Constitución recoge estos avances estableciendo en el artículo 360 que: La red pública integral de salud será parte del sistema nacional de salud y estará conformada por el conjunto articulado de establecimientos estatales, de la seguridad social y con otros proveedores que pertenecen al Estado ${ }^{(18)}$.

La conformación y el funcionamiento de la RPIS implican un avance en la construcción del SNS, que hasta el momento presenta algunos logros por destacarse.

Recuperación de rectoría del MSP. Sin duda, el marco normativo constitucional, sumado a la decisión política del más alto nivel, ha permitido que en el funcionamiento de la RPIS, esté claramente definido y se respete el rol de autoridad del MSP con la subordinación correspondiente de los otros actores de la red, tanto públicos como privados. Para el ejercicio de esta rectoría ha sido necesario el fortalecimiento institucional del MSP, con la creación del Viceministerio de Gobernanza y Vigilancia de la Salud, cuya Subsecretaria de Gobernanza, cuenta con una dirección nacional directamente responsable del funcionamiento 
de la RPIS, denominada Dirección Nacional de Articulación de la Red Pública y Complementaria de Salud. Además, cuenta con la Dirección Nacional de Políticas y Modelamiento del SNS, responsable de establecer el marco político para ese funcionamiento (19). Otro aspecto del avance de la RPIS ha sido, por primera vez en el país, la planificación conjunta de la oferta de servicios en base a necesidades de salud de acuerdo al perfil epidemiológico de un territorio con su adscripción poblacional definida. Esto implica que el usuario, independiente de su situación de afiliado o no a un seguro, pueda ser atendido por el prestador asignado cerca de su casa o si requiere referencia por el hospital de cualquiera de los prestadores que corresponda a la red de su territorio.

En el marco de este funcionamiento en red es necesario el pago de servicios entre prestadores, puesto que habrá usuarios asegurados que se atiendan en el MSP, o viceversa. En este sentido, otro logro ha significado la puesta en vigencia de un tarifario único de prestaciones, que, además, regula el pago en la compra de servicios a los proveedores privados ${ }^{200}$. Además del tarifario, el MSP ha fortalecido su rectoría avanzando en la normatización de otros aspectos del funcionamiento de la red, que van permitiendo el modelamiento del SNS. Entre ellos, la tipología y licenciamiento de las unidades de salud, la elaboración de las Guías de Práctica Clínica para condiciones de salud priorizadas, el proceso de definición del conjunto de prestaciones para una cartera única de servicios en cada nivel del sistema, los parámetros para la planificación conjunta de compras de medicamentos e insumos. Un logro significativo fue retomar el control de los servicios de recuperación de adicciones, muchos de los cuales, incluso, vulneraban derechos humanos fundamentales (21). Otro esfuerzo, todavía inicial, es la elaboración de Instrumentos para la gestión homologada de los sistemas de información.

\section{FORTALECIMIENTO DEL RECURSO HUMANO}

Una de las mayores preocupaciones en la reforma ha sido enfrentar el deterioro del recurso humano en la red pública, pero principalmente en el MSP. Deterioro caracterizado por grandes brechas en el número de profesionales necesarios, en todos los niveles del sistema, bajos salarios y ausencia de estabilidad laboral. Uno de los aspectos priorizados ha sido la formación del recurso humano. Cabe destacar la existencia al momento en formación de 1673 técnicos de atención primaria en salud (TAPS), 1128 profesionales cursando diferentes posgrados en el país y más de 100 fuera ${ }^{(12)}$. Con relación a los salarios, estos se han incrementado sustancialmente para todo el personal de salud. En el caso de los médicos, por ejemplo, ha implicado un aumento promedio de aproximadamente el $80 \%$ desde el comienzo del actual Gobierno (15). Por otro lado, la política laboral ha estado orientada a procurar una distribución más equitativa del recurso humano en el territorio nacional. Esto se ha conseguido con la homologación de salarios en el sector público en el marco de la Ley Orgánica del Servicio Público que ha frenado la migración de personal entre los prestadores públicos, y a través de la creación de incentivos salariales para zonas geográficas de difícil acceso ${ }^{(12,23)}$. Un programa digno de mención, es el llamado "Ecuador Saludable, Vuelvo por ti", que ha conseguido incorporar al 2013 a más de 760 profesionales de la salud promoviendo su retorno del exterior ${ }^{(11)}$.

\section{SISTEMA BASADO EN ATENCIÓN PRIMARIA EN SALUD: PRIORIZAR PREVENCIÓN Y PROMOCIÓN}

Si bien en un primer momento el cierre de brechas se enfocó en el ámbito hospitalario, siempre fue claro que la consolidación del SNS tiene que sustentarse en la APS. Por ello, la reorientación de la inversión pública en la infraestructura y el recurso humano de los servicios de salud hacia el primer nivel. En la planificación de la RPIS hasta el 2017, al estimar la suma de gasto corriente e inversión, se amplía significativamente el porcentaje para primer nivel de $35 \%$ en el 2013 para $51 \%$ en el 2017 , mientras que el de segundo y tercer nivel disminuye de $65 \%$ en el 2013 a $40 \%$ en el $2017^{(5)}$. Una parte significativa de la inversión en talento humano está orientada a fortalecer los equipos para el primer nivel de atención. Por un lado, la formación de los TAPS, que serán parte del equipo de primer nivel y cuya perfil técnico es para el trabajo de prevención y promoción, no para lo curativo. En el proceso de adscripción territorial y poblacional del primer nivel, se tiene previsto 2 TAPS por cada equipo de salud.

Por otra parte, está previsto que hasta el 2017 todos los profesionales obtengan la especialidad de salud familiar y comunitaria. En el caso del posgrado de medicina familiar y comunitaria su objetivo es elevar la capacidad resolutiva clínica del médico de primer nivel, en el marco del modelo de atención integral de salud (MAIS) (24). Para la implementación del posgrado, el MSP trabajó con nueve facultades de medicina a nivel nacional y, por primera vez en el país, se estructuró un currículo único basado en la necesidad de la red pública. Otro aspecto del fortalecimiento de la APS que es parte del MAIS y merece destacarse es la incorporación en el primer nivel de atención de los servicios de salud mental, rehabilitación y cuidados paliativos.

La otra vertiente de fortalecimiento de la APS desde la promoción de la salud, es el diseño institucional que 
permita extender la rectoría del MSP a los aspectos determinantes de la salud que dependen de las acciones de otros sectores o niveles de gobierno, tanto nacional como local. La posibilidad del MSP, como la autoridad sanitaria nacional, de actuar sobre los determinantes de la salud está sustentada en tres aspectos:

1. Capacidad institucional de generar, recoger $y$ sistematizar información pertinente para la toma de decisiones tanto dentro como fuera del sector. Para ello, ha sido necesario fortalecer institucionalmente al MSP en tres áreas: la generación de información epidemiológica y estadística, la creación de una Dirección Nacional de Economía de la Salud ${ }^{25)}$ y la creación del Instituto Nacional de Investigación en Salud Pública (26).

Este fortalecimiento ha permitido que el MSP pueda sustentar exitosamente la argumentación de la salud pública en la discusión de políticas públicas que intervienen sobre los determinantes de la salud. Por ejemplo, cuando se ha discutido con los ministerios del área de comercio y producción medidas como impuestos a la comida chatarra, alcohol y tabaco, o reglamentos como el de fijación de precios de medicamentos. Así como cuando se ha logrado posicionar con el Ministerio de Finanzas el criterio de salud pública al definir las prioridades de inversión del Estado en agua y saneamiento.

De hecho, en este trajín intersectorial del MSP, se han conseguido también otros importantes avances como el reglamento de etiquetado de alimentos ${ }^{(26)}$ y la inclusión del artículo 94 de la Ley de Comunicación que en su parte pertinente dice: Los medios de comunicación no podrán publicitar productos cuyo uso regular o recurrente produzca afectaciones a la salud de las personas, el Ministerio de Salud Publica elaborará el listado de estos productos. La publicidad de productos destinados a la alimentación y la salud deberá tener autorización previa del Ministerio de Salud Pública ${ }^{(27)}$.

2. Fortalecimiento institucional en la capacidad del MSP para regulación y control, resalta la creación de la Agencia para Regulación y Control Sanitario (ARCSA) y la propuesta para creación de la Agencia para Control de la Calidad de Servicios de Salud (ACESS), aún en discusión en el marco del Código Orgánico de la Salud.

En ARCSA, se ha invertido para fortalecer su eficiencia en la entrega de servicios a través de procesos informatizados y la actualización y modernización de su marco normativo, así como en la ampliación y renovación de sus laboratorios y procesos de control con fortalecimiento del recurso humano.

3. El tercer aspecto es garantizar el adecuado proceso de desconcentración, lo cual implica construir unidades territoriales desconcentradas con mayores posibilidades de planificación y desarrollo mejorando la capacidad resolutiva de los territorios, así como el nivel de interlocución apropiada con los gobiernos locales a la hora de definiciones de políticas que intervienen sobre los determinantes de la salud. Todo esto en el marco de la rectoría estatal de planificación para la desconcentración de todos los servicios públicos, en zonas, distritos y circuitos ${ }^{(28)}$.

\section{SOSTENIBILIDAD FINANCIERA}

Una de las preocupaciones permanentes del MSP y del gobierno ha sido la sostenibilidad financiera de este proceso de reforma, cuya discusión parte de reconocer que: la salud es un derecho que está garantizado por el Estado, por tanto, tienen que buscarse los mecanismos para asegurar su adecuado financiamiento; que el control de costos se garantizará con una oferta de servicios mayoritariamente pública, conjugado con fortalecimiento de la autoridad sanitaria nacional para un apropiado control hacia la eficiencia de los procesos en la entrega de servicios, así como en la generación de políticas que incidan sobre los determinantes de la salud, desde el interés público; que el SNS tiene que basarse en la APS, privilegiando los aspectos preventivos y promocionales; y que el financiamiento tiene que ser equitativo, solidario, sostenible y eficiente.

Para definir las necesidades de financiamiento, se calculó la cobertura para el 2013 en $75 \%$ de la población, correspondiendo aproximadamente $41 \%$ al MSP, $31 \%$ al IESS, y $3 \%$ a ISSFA e ISSPOL. Por otro lado, el gasto corriente de la RPIS en el 2013 fue de aproximadamente 3200 millones de USD, 49,7\% correspondiente al MSP y $50,3 \%$ a IESS, ISSFA e ISSPOL. Lo que significó para el MSP un per cápita de 240 USD y para IESS, ISSFA e ISSPOL de $302{ }^{(29)}$. La propuesta del MSP es aumentar la cobertura a un $15 \%$ adicional para el año 2017 con el conjunto de prestaciones establecido para la RPIS. De acuerdo con la estimación de costos de dichas prestaciones, realizada por el MSP, esto se podría lograr con un per cápita de 338 USD. El financiamiento provendría de las mismas fuentes que en el 2013, es decir, las cotizaciones para salud de los trabajadores afiliados a los seguros públicos (con la posibilidad de ampliación del número de afiliados debido a los procesos de formalización del trabajo, del 2007 al 2013 los afiliados subieron del 26\% de la 
población económicamente activa al $43 \%$ ) (30), así como del presupuesto fiscal del MSP. Con este per cápita, la proyección del gasto público de salud para el año 2017 sería de 7,3\% del PIB ${ }^{(31)}$. Este nivel de gasto, con la puesta en vigencia del conjunto de prestaciones para toda la RPIS, y la sustancial mejora de la calidad del servicio conseguida hasta el momento, permitirá garantizar un acceso efectivo, equitativo y de calidad para todo ciudadano, independiente de que sea o no afiliado a un seguro.

Estoimplica, necesariamente, diseñarunanuevaingeniería financiera para salud, que permita el funcionamiento de un fondo mancomunado para racionalizar el pago y transferencia de recursos a los prestadores, tomando en cuenta la adscripción territorial, incentivos financieros por calidad y cumplimiento de metas. En la práctica, esto permitirá equiparar el per cápita de los proveedores públicos. Si bien, esta nueva ingeniería financiera aún está en debate, hay algunas definiciones orientadas a mejorar la eficiencia del sistema, como el pago en primer nivel mediante capitación con cumplimiento de metas de salud pública (dilución de riesgo y contención de costos) y en base a adscripción territorial.

Por otro lado, tanto los procesos de fortalecimiento institucional como los nuevos marcos regulatorios, están orientados a la optimización y eficiencia de los recursos, mejorando la calidad del gasto. Entre estos se puede destacar los avances en los procesos de informatización, la planificación en base a necesidades de salud, organizada en red territorial y con adscripción territorial única de la población, privilegiando las intervenciones de mayor costo efectividad, como las de promoción de la salud y prevención de la enfermedad, así como también nuevas normativas como el reglamento para fijación de precios de medicamentos ${ }^{(30)}$ que implicará una disminución significativa global del precio de medicamentos en el mercado del país. En el mismo sentido, el fortalecimiento de ENFARMA (Empresa Pública de Fármacos) como proveedora de la RIPS incidirá también favorablemente para los precios en el mercado de medicamentos (28). Igualmente, todos los procesos en marcha orientados a mejorar la calidad de la atención redundaran en el control de costos de la atención.

\section{CONCLUSIONES}

La reforma de la salud en Ecuador es un proceso muy reciente y ha tenido importantes avances gracias a la decisión política para tratar de profundizarla en un corto plazo. Sin embargo, su sostenibilidad va a ser garantizada solamente cuando se convierta en una política de Estado que supere el margen de tiempo de un gobierno determinado. Para ello, consideramos que debe orientarse el esfuerzo para su consolidación en tres ámbitos relevantes:

Completar la reestructuración del marco jurídico, que en el caso ecuatoriano, significa la aprobación del nuevo Código Orgánico de la Salud, cuyo borrador recoge todo lo avanzado hasta el momento, así como aprobar las reformas pertinentes a la Ley de Seguridad Social, compatibilizando de una vez estas leyes, actualmente vigentes, con la Constitución de la República.

Profundizar el trabajo con el recurso humano de la RPIS para fortalecer los cambios actitudinales y las condiciones laborales que permitan revalorizar el servicio público hacia la generación de una cultura de calidad y eficiencia.

Ampliar y profundizar los mecanismos de participación ciudadana que permitan garantizar adecuados y eficientes niveles de control social en la gestión de la salud, pero sobre todo, que generen condiciones para que los ciudadanos se involucren críticamente en la discusión de la política y consideren, de ser pertinente, reivindicar ante cualquier gobierno los beneficios de una reforma de esta naturaleza.

Contribuciones de autoría: MM y NM han participado en la concepción del artículo, la recolección de datos, su redacción y aprobación de la versión final.

Conflictos de interés: los autores declaran no tener conflictos de interés.

Fuentes de financiamiento: autofinanciado. 


\section{REFERENCIAS BIBLIOGRÁFICAS}

1. Gudynas E. Buen Vivir: Today's tomorrow. Development. 2011;54(4):441-7.

2. Asamblea Nacional Constituyente de Ecuador. Constitución de la República del Ecuador. Registro Oficial 449 de 20 de octubre de 2008. Quito: Asamblea Nacional Constituyente de Ecuador; 2008.

3. World Health Organization. Health Promotion [Internet]. Ginebra: WHO; 2013 [citado el 17 de septiembre de 2014]. Disponible en: http:// www.who.int/healthpromotion/ conferences/8gchp/statement_2013/ en/

4. Secretaría Nacional de Planificación y Desarrollo. Secretaria Nacional de Planificación y Desarrollo [Internet]. Quito: SENPLADES; 2014 [Citado el 17 de septiembre de 2014]. Disponible en: http://www.planificacion. gob.ec/wp-content/uploads/ downloads/2014/04/folleto_7_esp_ web-2.pdf

5. Malo, M. Reforma del Sistema de Salud del Ecuador: hacia la cobertura universal [Internet]. Lima: MSP: 2014 [citado el 17 de septiembre de 2014]. Disponible en: http://www.paho.org/ forocoberturagt2014/wp-content/ uploads/2014/08/Reforma-delSistema-de-Salud-del-Ecuador-haciala-Cobertura-Universal.pdf

6. Instituto Nacional de Estadísticas y Censos. Pobreza por Ingresos ENEMDU. Resultados a Diciembre 2010 [Internet]. Quito: INEC; 2010 [citado el 17 de septiembre de 2014]. Disponible en: http://www.ecuadorencifras.gob. ec/documentos/web-inec/POBREZA/2010/dic-2010/Resul_Pobreza_ xIngr_NacUrRu_Dic2010.pdf

7. Instituto Nacional de Estadísticas y Censos. Reporte Economía Laboral. Diciembre 2013 [Internet]. Quito: INEC; 2013 [Citado el 17 de septiembre de 2014]. Disponible en: http://www.ecuadorencifras.gob.ec/documentos/ web-inec/EMPLEO/Informacion-2010-2011-2012-2013/2013/ Diciembre2013/15anios/Reporte_ Laboral-Diciembre_2013.pdf

8. Instituto Nacional de Estadísticas y Censos. Encuesta Nacional de Empleo, Desempleo y Subempleo - ENEMDU.
Indicadores de Pobreza y Desigualdad junio 2014 [Internet]. Quito: INEC; 2014 [citado el 17 de septiembre de 2014]. Disponible en: http://www. ecuadorencifras.gob.ec/documentos/web-inec/POBREZA/2014/Junio_2014/201406_EnemduPresentacion_Pobreza.pdf

9. Secretaria Nacional de Planificación y Desarrollo. Plan Nacional para el Buen Vivir 2013-2017 [Internet]. Quito: SENPLADES; 2013 [citado el 17 de septiembre de 2014]. Disponible en: http://documentos.senplades.gob. ec/Plan\%20Nacional\%20Buen\%20 Vivir\%202013-2017.pdf

10. Organización Panamericana de la Salud. Perfil de los Sistemas de Salud: Ecuador, monitoreo y análisis de los procesos de cambio y reforma. 3ra ed. [Internet]. Washington, D.C.: OPS; 2008 [citado el 22 de septiembre de 2014]. Disponible en: http://pdf. usaid.gov/pdf_docs/Pnadr438.pdf

11. Ministerio Coordinador de Desarrollo Social. Ministerio de Salud Pública. Rendición de cuentas 2013 [Internet]. Quito: MCDS; 2013 [citado el 26 de septiembre de 2014]. Disponible en http://www.salud.gob.ec/wp-content/ uploads/downloads/2014/05/ MSP2013_MCDS30demarzo.pdf

12. Servicio Integrado de Seguridad que conforman Sistema Nacional Salud. Decreto Ejecutivo № 988. Registro Oficial 618, Quito (13 enero, 2012).

13. Enlace Ciudadano. Gestión y sostenibilidad del Sistema Nacional de Salud [Internet]. Quito: SIGEF; 2013 [citado el 27 de septiembre de 2014]. Disponible en http://www.slideshare. net/fullscreen/PresidenciaEc/3sistema-de-salud-final-dan/1

14. Ministerio de Salud Pública. Ministerio de Salud Pública saluda al médico ecuatoriano por su noble labor [Internet]. Quito: MSP; 2014 [citado el 27 de septiembre de 2014]. Disponible en http://www.salud.gob.ec/ministeriode-salud-publica-saluda-al-medicoecuatoriano-por-su-noble-labor/

15. Ministerio de Salud Pública. Informe de Rendición de Cuentas 2013. Quito: MSP; 2014.

16. Ley Orgánica del Sistema Nacional de Salud. Ley No 80. Registro Oficial 670, (25 septiembre, 2002).
17. Ley Orgánica de Salud. Ley 67. Registro Oficial Suplemento 423, (22 diciembre, 2006).

18. Constitución de la República del Ecuador. Registro Oficial 449, (20 octubre, 2008).

19. Ministerio de Salud Pública. Organigrama del Ministerio [Internet]. Quito: MSP; 2014 [citado el 23 de septiembre de 2014]. Disponible en http://instituciones. msp.gob.ec/images/Documentos/ Ley_de_Transparencia/2014/abril/ organigrama_msp2014.pdf

20. Ministerio de Salud Pública. Tarifario de Prestaciones para el Sistema Nacional de Salud [Internet]. Quito: MSP; 2012 [citado el 17 de septiembre de 2014]. Disponible en http:// instituciones.msp.gob.ec/images/ Documentos/gobernanza/tarifario prestaciones_sistema_nacional_salud. pdf

21. Vance Mafla C. Reglamento de regulación de los centros de recuperación para tratamiento a personas con adicciones o dependencias a sustancias psicoactivas. Acuerdo Ministerial 767. Registro Oficial Suplemento 720 (08 junio, 2012).

22. Ley Orgánica de Servicio Público. Ley 0. Registro Oficial Suplemento 294 (06 octubre, 2010).

23. Norma para bonificación geográfica a servidores del sector salud. Acuerdo Ministerial 1. Registro Oficial 620 (1701-2012).

24. Ministerio de Salud Pública. Red Pública Integral de Salud. Manual del Modelo de Atención Integral de Salud - MAIS [Internet]. Quito: MSP; 2012 [citado el 25 de septiembre de 2014]. Disponible en http://instituciones. msp.gob.ec/somossalud/images/ documentos/guia/Manual_MAISMSP12.12.12.pdf

25. Agencia Nacional de Regulación Control y Vigilancia Sanitaria. Decreto Ejecutivo 1290. Registro Oficial Suplemento 788 (13 setiembre, 2012).

26. Agencia Nacional de Regulación Control y Vigilancia Sanitaria. Reglamento sanitario sustitutivo de etiquetado de alimentos procesados para consumo humano. Acuerdo Ministerial 5103. Registro Oficial Suplemente 318 (25 agosto, 2014). 
27. Registro Oficial. Órgano del Gobierno del Ecuador. Ley Orgánica de Comunicación. Registro Oficial No 22. Tercer Suplemento (25 junio, 2013).

28. Secretaría Nacional de Planificación y Desarrollo. Proceso de desconcentración del Ejecutivo en los niveles administrativos de planificación [Internet]. Quito: SENPLADES; 2012 [citado el 23 de septiembre de 2014]. Disponible en http://www.planificacion. gob.ec/wp-content/uploads/downloads/2012/10/Folleto_informativoDesconcentracion2012.pdf

29. Malo, M. Informe de estimaciones presupuestarias de salud para la Presidencia de la República; 2013 [Documento interno]. Quito: MSP; 2013.

30. Reglamento para la fijación de precios medicamentos de uso y consumo humano. Decreto Ejecutivo 400. Registro Oficial No 299. Segundo Suplemento (29 julio, 2014).
31. Creación de la empresa pública de fármacos, ENFARMA EP. Decreto Ejecutivo 181. Registro Oficial Suplemento 98 (30 diciembre, 2009).

Correspondencia: Miguel Malo-Serrano Dirección: Los Pinos 251, Urb. Camacho, La Molina, Lima

Teléfono: (51-1) 3195771

Correo electrónico miguel.maloserrano@ gmail.com

Visite nuestra página en Facebook, www.facebook.com/rpmesp Infórmese sobre los eventos y los nuevos contenidos de la Revista Peruana de Medicina Experimental y Salud Pública

\section{REVISTA PERUANA DE MEDICINA EXPERIMENTAL Y SALUD PÚBLICA} Difundiendo investigación en salud

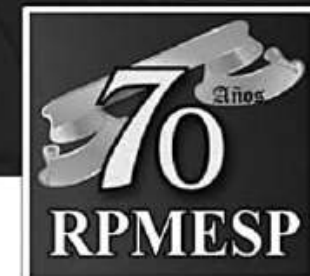

\section{$1942-2012$}

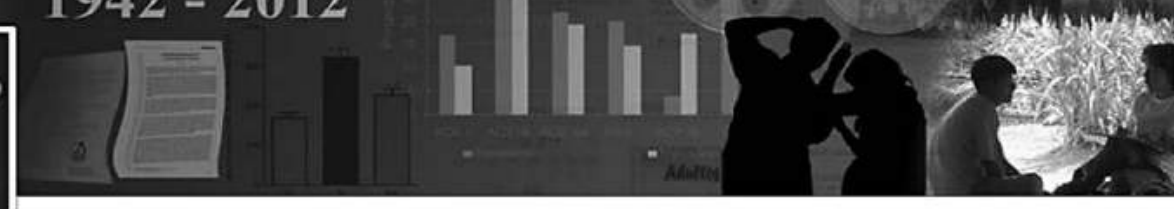

Revista Peruana de Medicina Experimental y Salud Pública (OFICIAL) A 1254 personas les gusta esta página $\cdot 8$ personas están hablando de esto

Comunidad

La Revista Peruana de Medicina Experimental y Salud Publica es el órgano oficial de difusión cientifica del Instituto Nacional de Salud

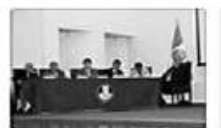

Fotos

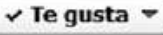

Siguiendo Mensaje 